\title{
Efecto de la probabilidad de error durante la acción de ataque en esgrima
}

\author{
C. GUTIÉRREZ-CRUZ, F.J. GILES, C. ZINGSEM, F.J. ROJAS, M. GUTIÉRREZ-DÁVILA \\ Departamento de Educación Física y Deportiva. Universidad de Granada
}

\begin{abstract}
Resumen
El propósito de este estudio ha sido comprobar el efecto que tiene la probabilidad de que surja el error durante la realización de una acción de ataque en esgrima, sobre los parámetros temporales de las respuestas de reacción (TRR) y la velocidad de desplazamiento del centro de masas (CM). Han participado 17 esgrimistas (14 hombres y 3 mujeres), con una experiencia en competición regional de más de cinco años. Para el registro de los datos, se han utilizado dos plataformas de fuerza, operando a $500 \mathrm{~Hz}$, una cámara de vídeo, a $210 \mathrm{~Hz}$ y un cronómetro electrónico adaptado al sistema de cableado de las armas. Una señal electrónica se utilizó para sincronizar temporalmente todos los registros. Para la situación experimental donde no existía incertidumbre durante la acción (CIA), los esgrimistas tenían que realizar un ataque simple con fondo lo más rápidamente posible al aparecer un círculo en el centro del plastrón (E1), intentando situar la punta de la espada dentro del círculo. Para la situación experimental donde existía incertidumbre debida a la posibilidad de error (CIA), los esgrimistas tenían los mismos objetivos que en la situación anterior, aunque debian de inhibir la acción de ataque a otra de defensiva, en el caso de que el círculo desapareciera del plastrón durante la acción. Los resultados ponen de manifiesto que, cuando existe la posibilidad de error durante la realización del ataque en esgrima, se incrementan los parámetros temporales de la respuesta de reacción (TR y TM) y disminuye la velocidad de ejecución.
\end{abstract}

Palabras clave: Control Motor, Biomecánica, Esgrima, Respuesta de reacción, Cambio de patrón de movimiento.

\begin{abstract}
The main aim of this study has been to verify the effect that has the probability from which the mistake arises during the accomplishment of an action of assault in fencing, on the temporary parameters of the answers of reaction (TRR) and the speed of displacement of the center of masses (CM). 17 fencers (14 men and 3 women), with an experience in regional competition of more than five years took part in this study. Two force platforms, operating to $500 \mathrm{~Hz}$ have been in used, a video camera, to $210 \mathrm{~Hz}$ and an electronic chronometer adapted to the system of wired up of the weapon for the record of the data. An electronic sign was used for synchronizing temporarily all the records. For the experimental situation where uncertainty did not exist during the action (CIA), the fencers had to realize a simple assault with lunge as rapidly as possible when a circle appeared in the center of the plastron (E1), trying to place the top of the sword inside the circle. For the experimental situation where there existed uncertainty due to the possibility of mistake (CIA), the fencers had the same aims that in the previous situation, though they must disable the action of assault to other one of defensive, in case the circle was disappearing of the plastron during the action. The results reveal that, when the possibility of mistake exists during the accomplishment of the assault in fencing, there are increased the temporary parameters of the response of reaction (TR and TM) and it diminishes the speed of execution.
\end{abstract}

Keywords: Motor Control, Biomechanics, Fencing, Reaction response, Change of movement pattern.

Correspondencia:

F.Javier Rojas Ruiz

Facultad de Ciencias de la Actividad Física y del Deporte. Departamento de Educación Física

Ctra. Alfacar s/n 18011- Granada, (España).E-mail: fjrojas@ugr.es 


\section{Introducción}

En esgrima, el éxito de la mayoría de las acciones de ataque a blancos lejanos, requieren la participación de un componente táctico que facilite la programación de un patrón de movimiento adecuado para engañar al oponente en su acción decuado para engan acción ngañar al oponente para tener exito en este tipo de acciones, se pone de manifesto a conprobar que los tienpos de movimiento utilizados por esgrimistas de alto nivel son 2.5 superiores al tiempo de reacción de elección requerido para iniciar la defensa [1] Así, el componente táctico, permite al esgrimista la predicción de las acciones defensivas que realizará el oponente, además de facilitar la programación de un patrón de movimiento adecuado para anticiparse a las acciones defensivas. Según lo expuesto, el concepto de anticipación para este tipo de acciones, est́ relacionado con la probabi-

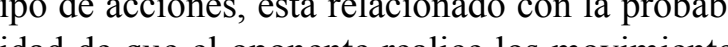
lidad de que el oponente realice los movimiento defensivos previstos por el atacante. Cuando esto no ocurre, surge el error y la necesidad de inhibir el patrón de movimiento inicial y cambiar a otro más adecuado.

El proceso de cambio de un patrón de movimiento a otro más adecuado, requiere un tiempo de procesamiento de la información, el cual estară condicionado por el nivel de incertidumbre creado por la acción no prevista del oponente en su de

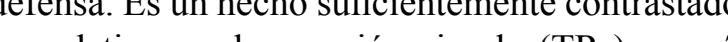
que el tiempo de reacción simple $\left(\mathrm{TR}_{\mathrm{S}}\right.$ ) es más reducido que el tiempo de reacción de elección (TRE) el cual se incrementa de forma logarítmica en función del número de respuestas posibles [2, 3]. En este sentido, Borysiuk y Waskiewicz [4] y Czajkowski [5] han puesto de manifiesto que la reducción de la incertidumbre en esgrimistas permite procesar la información más rápidamente $y$, por consiguiente, responder con mayor rapidez y ficiencia. Gutiérrez-Dávila, Rojas, Antonio y Navarro han constatado que el TRE durante las acciones de ataque, en esgistas de alto lav acciones de ataque, en esgrinistas de alto nivel, se inceriando el número de respuestas posibles pasa de dos a cuatro [6].

Con el propósito de reducir la incertidumbre, cuando surge el error durante la acción de ataque la respuesta alternativa suele asociarse a un patrón de movimiento programado y no a la acción imprevista del oponente. Es decir, a cada patrón de movimiento programado para el ataque, se le asociaría una sola acción alternativa en el caso de producirse el error, de este modo el número de res- puestas se reduce a una. Según lo expuesto, la incertidumbre durante la acción de ataque, está condicionada por el nivel de probabilidad de error que tiene el esgrimista antes de realizarse la acción, lo que constituye una intención de movimiento consciente que puede verse influenciada por este tipo de incertidumbre [7].

Para garantizar la adaptabilidad en la respuesa cuando existe incertidumbre durante la acción se necesita una facilitación estratégica o de alto orden, que permita inhibir la primera acción que desencadena un estímulo para aumentar la precisión de la respuesta [8]. Siguiendo a Duque, Lew, Mazzocchio, Oliver y Ivry [9] este proceso de inhibición de respuestas se desarrolla mediante dos mecanismos estrechamente relacionados: El primero inhibe la activación de las posibles respuestas seleccionadas a nivel espinal (control de impulsos), con el propósto de evitar acciones preme presción Aś́ as de inos (resolicion de conthictos). Asi, antes de iniciar el movimiento, se activarían todas las respuestas posibles, requiriéndose señales inhibitorias a nivel espinal, a la espera de información exterior que haga prevalecer una de ellas. Este segundo mecanismo inhibitorio ocurriría a niveles corticales superiores, produciendo una cierta demora en la respuesta [10,11], lo que nos permite pensar que la incertidumbre debida la posibilidad de error durante la acción de ataque, (a) crementaria el TR de los esgrimistas y la veloci-

La influencia que tiene este tipo de incertidumbre, provocada por la previsión de error durante 1 acción, también podría explicarse con el model teórico sobre las dos corrientes de la percepción visual $[12,13]$. A partir de este modelo, la respuesta de reaccion requiere la contribución de dos procesos visuales que tienen funciones diferentes. La corriente o sistema dorsal (dirigida, desde la corteza visual primaria, hacia el lóbulo parietal posterior), se encargaría de identifcar la localización de los objes y sus movimientos, recoge la informacos a ción visual inplicita en el control del movimiento, inmediata y relativamente rápila. La corriente sistema ventral (dirigida, desde la corteza visua primaria, hacia la corteza del lóbulo temporal inferior), asociada a la consciencia explícita, estarí encargada de recoger la identidad de los objetos, informando sobre las probabilidades que existen para llevar a cabo la acción.

A pesar de las diferencias expuestas, los dos sistemas trabajan en estrecha colaboración $[14,15]$ aunque, dependiendo de la situación, el control del movimiento puede estar dominado más por una que otra corriente visual. Así, cuando se realiza una acción sin incertidumbre durante la acción la corriente ventral dominaría antes de iniciase el movimiento, mientras que durante la acción sería la corriente dorsal quien dominaría sobre la ventral, favoreciendo la rapidez del movimiento. En cambio, cuando existe incertidumbre durante

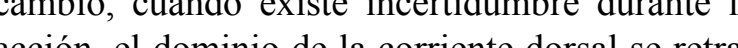
cion, etrasaria hasta el instante en que se produce el cambio de acción, lo que podría contibuir a que el desplazamiento hasta ese instante fuese más lento.

Según lo expuesto, el propósito de este estudi ha sido comprobar el efecto que tiene la probabilidad de que surja el error durante la realización de un patrón de movimiento (nivel de incertidumbre), sobre los parámetros temporales de las respuestas de reacción (TRR) y la velocidad de desplazamiento del centro de masas (CM) durante late con fondo de eserima. Con el proposito e elinina la incetide de eliminar la incentidunbre debida al número de respuestas asocis al duce, deberá inhibirse el patron de movimiento inicial para cambiar a otro alternativo asociado a primero. Según lo expuesto, consideramos como hipótesis que la incertidumbre debida a la probabilidad de error durante la acción, incrementará e tiempo de reacción al estímulo $\left(\mathrm{TR}_{1}\right)$ y reducirá la velocidad de desplazamiento del $\mathrm{CM}$.

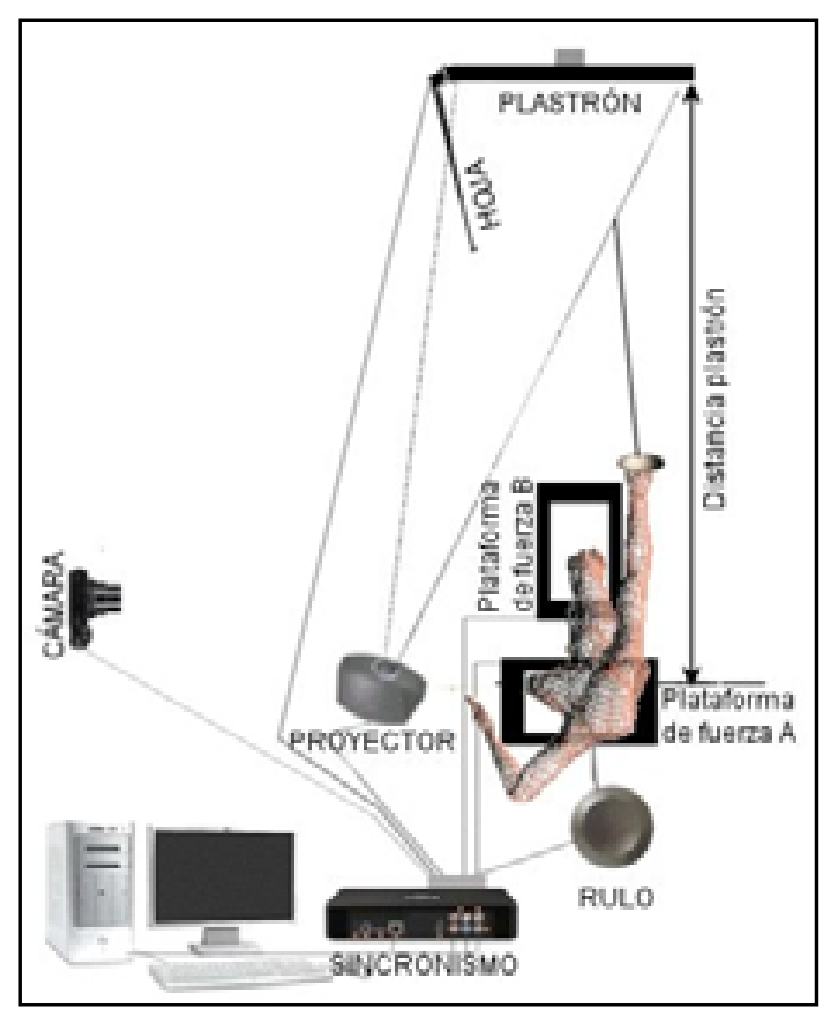

Figura 1. Representación esquemática del protocolo y los sistemas de registro utilizados.
Han participado 17 esgrimistas (14 hombres y 3 mujeres), con una experiencia en competición regional de más de cinco años. Diez de ellos eran especialistas en espada y el resto eran especialistas en florete (edad $=25.6 \pm 6.5$ años; talla $=1.75 \pm 0.08$ m. mass $=70 \pm 11.1 \mathrm{Kg})$. A todos ellos se les inforof $11.1 \mathrm{Kg}$ ) A todos ellos se les inforeste stio sigien las directices de la Conisón Ética de la Universidad.

Se han utilizado dos plataformas de fuerza de 0.6 x 0.37 m, Dinascan/IBV, (Instituto de Biomecánica de Valencia, Valencia, España), operando $500 \mathrm{~Hz}$, las cuales registraban la componente horizontal de la fuerza de reacción $\left(\mathrm{F}_{\mathrm{AX}}\right.$ y $\left.\mathrm{F}_{\mathrm{BX}}\right)$. Una camara de vídeo (Casio EX - FH20, Tokio, Japón) $210 \mathrm{~Hz}$ registraba el plano sagital de los esgrimistas. Un proyector asociado a un ordenador con arjeta externa programable, permitía el control proyección de un círculo negro de $0.09 \mathrm{~m}$ de dínetro, sobre el cento geon de una pantla blanca de $0.70 \times 0.55 \mathrm{~m}$, que tuaba co a pastrón. En uno de los laterales de 0.55 menpentento una hoja de espada de . $55 \mathrm{~m}$, perpendicular al plano. Las armas persoeronometro electrónico (1/1000 s), adaptado al sistema de caleado, que registraba el tiempo de la respuesta de 
reacción (TRR). Una señal electrónica se utilizó para iniciar los registros de las dos plataformas y el cronómetro. Esta misma señal se utilizó para e sincronismo temporal de la cámara, a través del encendido de un LED situado en su campo visual. En la figura 1, se presenta un esquema de los equipos utilizados.

Tras un calentamiento previo de 15 minutos, se ajustó la altura del plastrón para cada esgrimista, situándose su centro geométrico al $75 \%$ de la talla. A continuación, recibieron las instrucciones de mantenerse quietos en su posición habitual de "en guardia" sobre las dos plataformas, situando e primer dedo del pie más retrasado a una distancia de 1.5 veces su talla, con respecto al plastrón. A aparecer el círculo proyectado en el plastrón $\left(\mathrm{E}_{1}\right)$ debían de realizar un ataque directo lo más rápido posible, situando la punta de la espada dentro del círculo. Después de realizar varias accio distancia preestablecida, se permitió que los esgrimistas realizaran los ajustes de distancia necesarios hasta que se sintieron cómodos en la nueva distancia. Los participantes redujeron la distancia inicial del plastrón una media de $0,002 \pm 0,07 \mathrm{~m}$. Antes de comenzar los ensayos de registro, los esgrimistas realizaron una sesión de varios ataques al plastrón hasta que se habituaron con el sistema.

Para los registros de la situación experimental donde no existía incertidumbre durante la acción (SIA), los esgrimistas partían desde la posición de "en guardia" y tras una señal previa que iniciaba un pre-periodo comprendido entre $1.5 \mathrm{~s}$ y $3.0 \mathrm{~s}$, enían que realizar un ataque simple con fondo lo más rápidamente posible al aparecer el círculo en el centro del plastrón $\left(E_{1}\right)$, intentando situar la punta de la espada dentro del círculo. Se realizaron cinco ensayos válidos para todos los sujetos en los que se registró el tiempo de la respuesta de reacción $\left(\mathrm{TRR}_{\mathrm{SI}}\right)$, considerado como el periodo de tiempo comprendido entre el instante en que aparece $E_{1}\left(t_{1}\right)$ y el contacto de la punta del arma col

Para los ensayos donde existía incertidumbre debida a la posibilidad de error (CIA), los esgrimisas tenían los mismos objetivos que en la situación anterior, aunque para esta situación experimental, tambien recibieron las instrucciones de inhibir la acción de ataque a otra de defensiva, en el caso de que el círculo desapareciera del plastrón durante la acción. El movimiento de defensa, alternativo a la acción de ataque, consistía en golpear una espad situada en un lateral del plastrón (lado del brazo no armado del esgrimista), realizando una acción de parada "de cuarta". Para evitar el factor aprendizaje, se realizaron 10 ensayos donde se prese taron de forma aleatoria las dos condiciones. cinco ensayos válidos donde desaparecía el círculo durante la acción de ataque y cinco ensayos donde el círculo se mantenía proyectado hasta que punta de la espada impactaba en el plastrón. Sólo e registraron los cinco ensayos válidos d pere re de esgnion de acció de la respuesta de reacción (TRR como como el periodo de tiempo comprendido entre $t_{1} y$ el instante en que la espada tomaba contacto con la hoja implementada al plastrón (contacto), analizándose sólo el ensayo donde el TRR $\mathrm{CIA}_{\mathrm{s}}$ obtení el registro mediano de los cinco registros válidos.

Las imágenes de vídeo fueron utilizadas para determinar la posición del CM del esgrimista más el arma antes del inicio del movimiento. Para ello, se digitalizaron cinco imégenes a una fecurencia de $30 \mathrm{~Hz}$, a parir de t, uilizando a modelo coordinado de catore segmentos más dos meloco situados en la hoja del arma (final del tecio fueste ju to a la puna ju ju (hinal del tercio fuerte y junto a la punta), junto a los parámetros inerciales propuestos por Zatsiorsky y Seluyanov [16] adaptados por de Leva [17]. Para la conversión de los datos a escala real se utilizó un sistema de referencias de 1.58x1.58x1 m. A partir del inicio de movimiento $\left(\mathrm{t}_{\mathrm{NNI}}\right)$, sólo se digitalizaron tres punto del sistema (los dos marcadores asociados al arma ex extemo posterior del pie más adelantad), a una frecuencia de $210 \mathrm{~Hz}$. Tras la conversión a escala rell las coordens planas de los tres ma cadores fueron filtrads utilizando un filtro digit de paso bajo a $8 \mathrm{~Hz}$ [18] e interpolados a $500 \mathrm{~Hz}$ utilizando splines de quinto orden.

A partir de los datos procedentes de las plataformas de fuerza, se determinaron los registro relativos a las velocidades y desplazamientos de CM del esgrimista más el arma utilizada. Para ello, se calculó la aceleración horizontal del CM plataformas $\left(\mathrm{F}_{\mathrm{AX}}\right.$ y $\left.\mathrm{F}_{\mathrm{BX}}\right)$ y la masa del esgrimista. A continuación, se determinó la componente horizontal de la velocidad $\left(\mathrm{v}_{\mathrm{x}(\mathrm{CM})}\right)$ y el desplazamiento del $\mathrm{CM}\left(\mathrm{s}_{\left.\mathrm{X}_{(\mathrm{CM})}\right)}\right)$, mediante la integración de la función aceleración-tiempo, usando par ello el método trapezoidal. Las constantes de integración se determinaron a partir de la media de las cinco posiciones del CM obtenidas a través de las imágenes de vídeo.

El inicio del movimiento $\left(\mathrm{t}_{\mathrm{INI}}\right)$, se determinó partir del instante en que la fuerza neta de compo- mayor o igual al $1 \%$ del peso del cuerpo. Cuando ese tiempo era inferior a $100 \mathrm{~ms}$, se repetía el ensayo. Para determinar el instante de cambio de acción $\left(\mathrm{t}_{\mathrm{CAM}}\right)$, se ha utilizado la componente vertical de la aceleración del marcador situado al final de tercio fuerte de la espada, utilizando para ello la segunda derivada de la función splines corresponiente a la posición vertical. sente a la posicion vertical. Anque el marcador situado en la punta de la espada era más sensible can que los movimientos debidos a la elasticidad de arma podrían producir ciertas interferencias.

Además de la respuesta de reacción, para cada situación experimental, se han registrado sus componentes temporales más significativas para este estudio:

a) tiempo de reacción de inicio del movimiento $\left(\mathrm{TR}_{1}\right)$, definido como el periodo comprendido entre la aparición del estímulo $\left(\mathrm{t}_{1}\right)$ y el inicio del movimiento $\left(\mathrm{t}_{\mathrm{INI}}\right)$ y

b) tiempo de movimiento (TM), definido como el periodo temporal comprendido entre $t_{\mathrm{IN} \mathrm{I}} \mathrm{y}$ el instante del contacto de la punta del arma con el plastrón (tocado) o el contacto con la hoja asociada al plastrón (contacto), para SIA o CIA, respectivamente.

Para la situación experimental donde se produce el cambio de acción (CIA), el tiempo de movimiento se ha dividido en:

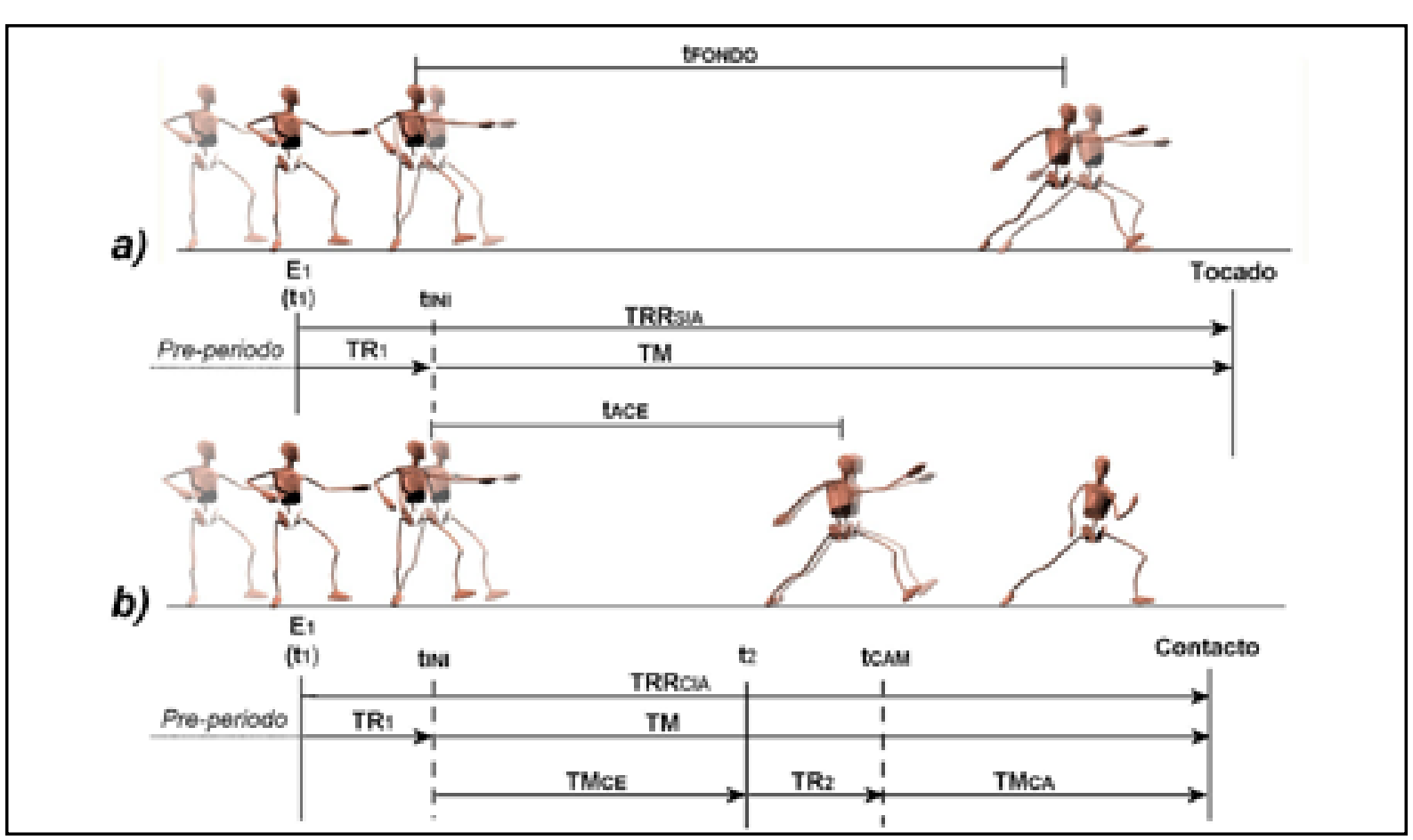

Figura 2. Representación esquemática de la secuencia de ataque simple junto a las fases del movimiento (superior), así como un modelo temporal simplificado del ataque simple ejecu-
tado con golpe recto (A) y del ataque simple con cambio en el patrón de movimiento (B). $\left.b_{1}\right)$ tiempo de movimiento al cambio de estímulo $\left(\mathrm{TM}_{\mathrm{CE}}\right.$ ), definido como el periodo compren-

$\mathrm{b}_{2}$ ) tiempo de reacción al cambio de estímulo $\left(\mathrm{TR}_{2}\right)$, definido como el periodo comprendido entre $t_{2} y$ el in acción $\left(\mathrm{t}_{\mathrm{CAM}}\right) \mathrm{y}$

$b_{3}$ ) tiempo de movimiento al cambio de acción (TMCA), comprendido entre $t_{\mathrm{CAM}} \mathrm{y}$ el instante $\mathrm{de}$ contacto con el arma en la hoja asociada al plasón (contacto).

unsarecia el circulo $\left(t_{2}\right)$, sea utilizado el TRR SIA $_{\text {mediano }}$ $\left.\left.\mathrm{TR}_{1}\right) / 2\right]$

Finalmente, el análisis temporal se ha completado con el tiempo que dura la fase de aceleración horizontal $\left(\mathrm{t}_{\mathrm{ACE}}\right)$, considerado como el periodo comprendido desde $t_{\mathrm{IN}}$, hasta que la fuerza horizontal resultante $\left(\mathrm{F}_{\mathrm{AX}}+\mathrm{F}_{\mathrm{BX}}\right)$ se hace inferior al $1 \%$ del peso del esgrimista.

En la figura 2 se representan las componente temporales de la respuesta de reacción para la condiciones SIA (a) y CIA (b). En la parte supeior se han representado las fases temporales de as secuencias de ataque con fondo, para las dos condiciones. dido desde $t_{\mathrm{INI}} \mathrm{y}$ el instante en que desaparece el 
Para completar el análisis, se ha registrado el espacio horizontal recorrido en el menor de los dos tiempos registrados para la fase de aceleración horizontal $\left(\mathrm{t}_{\mathrm{ACE}}\right)$, de cada esgrimista $\left(\mathrm{s}_{\mathrm{X}(\mathrm{CM})}\right.$ truncado), así como la velocidad horizontal al final de ese mismo periodo ( $\mathrm{v}_{\mathrm{x}_{(\mathrm{CM})}}$ truncado). Finalmente, a partir de las imágenes de vídeo, se han registraa dos vables: a) el tiempo utilzado pera reat-

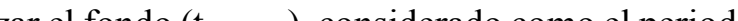
empor conprendido entre ty ed insate en el marcador del talón toma col to $\mathrm{y}$ b) la longitud del fondo ( $\mathrm{s}_{\mathrm{FONDO}}$ ), definida como la distancia comprendida entre el marcador de la punta del pie más retrasado en la posición de guardia y el marcador situado en el talón del pie más adelantado cuando está plenamente apoyado en el suelo después de realizar el fondo.

Para el tratamiento estadístico de los datos se ha utilizado el software Statgraphics 5.1 de Stadistical Graphics Corporation (STCS Inc, Rockville, MD). Para cada variable y situación experimental se ha calculado la media y desviación típica. Para determinar las diferencias entre las medias de las variables en las dos situaciones experimentales (SIA y CIA, respectivamente), se ha aplicado un análisis de varianza multifactorial para medidas repetidas (ANOVA). Para evaluar la fiabilidad de las pruebas, se aplicó un análisis de varianza de medidas repetidas a todos los ensayos en las dos condiciones experimentales (cinco ensayos), omand como varible dependiente el tienpo

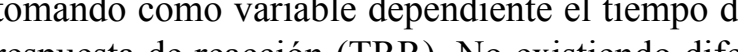
respuesta de ieacción (TRR). No existiendo diferencias significativas entre los ensayos. El coeficiente de correlación intraclase ha sido de 0.914 $(\mathrm{p}<0.001)$ para la situación de ataque con golpe recto sin incertidumbre (SIA) y de 0.866 ( $p$ 0.001) para la situación con incertidumbre debida la posibilidad de error durante la acción (CIA)

\section{Resultados}

En la tabla 1 se presenta la estadística descriptiva y las diferencias entre las medias de las variables más significativas, para las dos condiciones de ataque con fondo propuestas:

a) sin incertidumbre durante la acción (SIA) y

b) con incertidumbre debida a la posibilidad de error (CIA).

Los datos ponen de manifiesto que la posición de "en guardia", antes de iniciarse el movimiento, es similar para las dos condiciones propuestas. Sin embargo, el tiempo de reacción (TR, ha sido significativamente menor cuando no existe incertidumbre $(\mathrm{p}<0.05)$.
A continuación, en la tabla 1, se presentan los datos para el instante en que desaparece el objetivo en la situación CIA $\left(\mathrm{t}_{2}\right)$. Aunque en la situació SIA no desaparezca el objetivo, se han registrado los valores para ese mismo instante. Los datos reflejan que existen diferencias significativas entre las medias $(\mathrm{p}<0.001)$, para el espacio horizontal recorrido

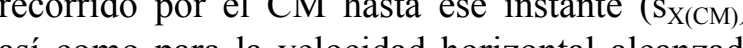
asi con ext $t_{2}\left(v_{x}(\mathrm{CM})\right)$. Según las condiciones experimentales, el tiempo de reacción para realizar el cambio de acción $\left(\mathrm{TR}_{2}\right)$, sólo existe en la situación CIA. Los registros medios sólo se presentan como una referencia con respecto a $\mathrm{TR}_{1}$. Así, han existido diferencias significativas cuando se comparan las medias de $\mathrm{TR}_{2}$ con las registradas para $\mathrm{TR}_{1}$ en la situación SIA ( 0.174 vs. $0.208 ; p<0.01)$, mientra que no han existido diferencias estadísticas cuando se compara con las media de $\mathrm{TR}_{1}$ en la situación CIA (0.198 vs. 0.208 s). Considerulo ate los sistemas de registro han sido diferentes para $\mathrm{TR}_{1} \mathrm{TR}_{2}$, deberím tación de estos resultados.

Como se esperaba, al ser la distancia al plastron igual para las dos condiciones (SIA y CIA, respectivamente), la distancia horizontal recorrida por el CM, ha sido similar en las dos situacione experimentales, aunque se ha requerido más tiempo, $\mathrm{t}(\mathrm{ACE})$ con la condición CIA ( 0.523 vs 0.558 ; $<0.05$ ). La velocidad horizontal media, al fin

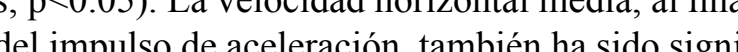
ficativan de ar vs. $1.65 \mathrm{~ms} 1 ; \mathrm{p}<0.05)$. Quizás los datos 1.76 presentativos de esta investigación se presento la tabla 1 como valores de tiempos igresentan en como valores de tiempos igualados en la fase de aceleración, donde se trunca el tiempo de mayor para hacerlo igual al menor. Con este termino se ha pretendido reflejar el bloqueo del factor tiempo al final de la fase de aceleración, lo que nos permite comparar el espacio recorrido por el CM de cada esgrimista, en el menor los dos tiempos de impulso de acelercionde las conliciones propuctas (s peloci(v horizontal alcanza $\left(\mathrm{V}_{\mathrm{X}(\mathrm{CM})}\right.$-truncado). Los datos ponen de manifiesto que, para las dos variables, existen claras diferencias significativas entre las medias $(\mathrm{p}<0.001)$. E decir, el espacio recorrido por el CM para un mismo tiempo, ha sido mayor cuando no existe incertidumbre, SIA, (0.370 vs. $0.294 \mathrm{~m}$ ), así como la velocidad registrada en ese mismo tiempo (1.74 vs $\left.1.52 \mathrm{~ms}^{-1}\right)$.

Finalmente, en la tabla 1, se presentan los datos asociados al instante en que la punta de la es- pada toma contacto con el plastron (tocado), para la situación SIA y el instante en que la hoja del arma toma contacto con la hoja asociada al plastrón (contacto), para la situación CIA. Los datos indican que el tiempo de la respuesta de reacción (TRR) y el tiempo de movimiento (TM), han sido ignificativamente mayores cuando existe incertigen posibilidad de error durante a acción (p<0.001). Según las condiciones propuestas, el tiempo de movimiento para realizar el cambio de acción, $\mathrm{t}_{\mathrm{CAM}}$, , sólo puede existir en la condición CIA, por lo que sus datos de tendencia central se presentan como un posible indicador de incremento de la respuesta de reaccion registrado para esta situación. Aunque la longitud media de fondo, SFONDo, ha sido similar para las dos condiciones, el tiempo utilizado para realizarlo ha sido significativamente mayor en CIA (0.642 vs. 0.713 $\mathrm{s} ; \mathrm{p}<0.05)$

\begin{tabular}{|c|c|c|c|}
\hline Variables & SIA & CIA & $\boldsymbol{F}$ \\
\hline \multicolumn{4}{|c|}{ Posición inicial "en guardia" ( $\left.t_{N}\right)$} \\
\hline Posición vertical $C M(m)$ & $0.895 \pm 0.072$ & $0.896 \pm 0.081$ & 0.00 \\
\hline Posición horizontal CM (m) & $0.356 \pm 0.083$ & $0.359 \pm 0.090$ & 0.05 \\
\hline Posición vertical cazoleta $(m)$ & $1.121 \pm 0.071$ & $1.102 \pm 0.072$ & 2.51 \\
\hline $\begin{array}{l}\text { Posición horizontal cazoleta } \\
(m)\end{array}$ & $0.986 \pm 0.011$ & $0.996 \pm 0.012$ & 0.50 \\
\hline$T R_{1}(s)$ & $0.174 \pm 0.017$ & $0.198 \pm 0.035$ & $6.54^{*}$ \\
\hline \multicolumn{4}{|c|}{ Cambio de estímulo $\left(t_{2}\right)$} \\
\hline $\boldsymbol{t}_{\text {(CAMBio estimulo) }}(s)$ & $0.451 \pm 0.030$ & $0.451 \pm 0.030$ & Cond. \\
\hline$s_{\mathrm{X}(\mathrm{CM})}(m)$ & $0.070 \pm 0.018$ & $0.048 \pm 0,025$ & $13.29^{* \star}$ \\
\hline$v_{\mathrm{X}(\mathrm{CM})}\left(m s^{-1}\right)$ & $0.730 \pm 0.112$ & $0.533 \pm 0,212$ & $14.92^{\star \star}$ \\
\hline$T R_{2}(s)$ & - & $0.208 \pm 36$ & - \\
\hline \multicolumn{4}{|c|}{ Final impulso de aceleración } \\
\hline$t_{(A C E)}(s)$ & $0.523 \pm 0.052$ & $0.558 \pm 0.084$ & $5.15^{*}$ \\
\hline$s_{X(C M)}(m)$ & $0.405 \pm 0.089$ & $0.386 \pm 0.097$ & 0.70 \\
\hline $\boldsymbol{v}_{X(C M)}\left(m s^{-1}\right)$ & $1.757 \pm 0.256$ & $1.650 \pm 0.257$ & $6.60^{*}$ \\
\hline \multicolumn{4}{|c|}{ Tiempos del impulsos de aceleración igualados } \\
\hline $\boldsymbol{s}_{\mathrm{X}(\mathrm{CM})}$-truncado $(m)$ & $0.370 \pm 0.087$ & $0.294 \pm 0.080$ & $21.11^{* * *}$ \\
\hline$v_{\mathrm{x}(\mathrm{CM})^{-}}$-truncado $\left(\mathrm{ms}^{-1}\right)$ & $1.742 \pm 0.256$ & $1.521 \pm 0.250$ & $20.97^{* \star *}$ \\
\hline \multicolumn{4}{|c|}{ Tocado / contacto } \\
\hline $\operatorname{TRR}(\mathrm{s})$ & $0.717 \pm 0.053$ & $0.795 \pm 0.067$ & $75.23^{\star \star \star}$ \\
\hline$T M(s)$ & $0.543 \pm 0.052$ & $0.601 \pm 0.060$ & $31.27^{* \star *}$ \\
\hline$T M_{\mathrm{CA}}(\mathrm{s})$ & - & $0.101 \pm 0.034$ & - \\
\hline$t_{\text {FONDO }}(s)$ & $0.642 \pm 0.060$ & $0.713 \pm 0.088$ & $6.42^{*}$ \\
\hline$s_{\text {FONDO }}(m)$ & $1.25 \pm 0.13$ & $1.30 \pm 0.27$ & 0.92 \\
\hline
\end{tabular}

Tabla 1. Estadística descriptiva y ANOVA para medidas repetidas de las variables más significasin incertidumbre durante la acción (SIA) y b) con incertidumbre debida a la posibilidad de error (CIA) 


\section{Discusión}

En esta investigación se ha puesto de manifiesto que, cuando existe incertidumbre debida a posibilidad de error durante la realización del ataque en esgrima, el tiempo de la respuesta de reacción es mayor que en ausencia de incertidumbre, incrementándose el tiempo de reacción (TR) y el tiempo de movimiento (TM). Considerando que la posición de partida (en guardia) ha sido la misma para las dos condiciones, se constata que a velocidad media de desplazamiento horizontal del CM, se reduce con la incertidumbre. Como se esperaba, debido a que la distancia al plastron ha sido la misma para las dos condiciones, no han existido diferencias significativas en el desplazamiento horizontal del CM durante el impulso de celeración, ni en la longitud media del fondo, aunque el tiempo utizzop pasu jecucion cen e rere variables. En consecteria, cundo existe incertidumbre debida a la posibilidad de error durante la acción, el movimiento se hace más lento, lo que confirma los resultados de Sanderson [19], Borysiuk y Waskiewicz [4] y Gutiérrez-Dávila et al [6]

Aunque se conocía la respuesta que debian de realizar en caso de error, sólo la posibilidad de que pueda existir el error durante la acción de ataque, ha provocado que la ejecución del movimiento haya sido más lenta, lo que confirma las teoría ropuestas por la neupsicologia cogitiva sobre propuestas por la ne, lo que contog cogitiva sobre Á, culo no by Así, cuando no hay prevision de error durante la acción (sin incertidumbre), los planes motores y su secuenciación surge mediante un patrón de sólo beneficios, conocido como facilitación automática. En este patrón el movimiento se desarrolla de forma implícita, inconsciente y automatizada, lo que constituye una ventaja para realizar respuestas rápidas. Sin embargo, cuando existe una previsión de que pueda producirse el error durante la acción, para garantizar la flexibilidad en las acciones de pambio de objetivo, el esgrimista co de tipo de facilltación, conocida cono facilitación estratégica o de alto orden, que permitiría al esgrimista inhibir la primera acción de ataque y cambia a otra acción asociada manteniendo la precisión de la respuesta, lo que justificaría el hecho de que el movimiento se realice más lentamente cuando existe la previsión de error durante el ataque.

En las dos situaciones existe una sola respuesta ante un mismo estímulo, es decir, el tiempo de reacción es simple y la respuesta es la misma para las dos condiciones, por lo que la explicación al incremento del TR ante la posibilidad de error, no puestas posibles ante un mismo estímulo [2, 3 . A este hecho, se le podría dar el mismo sentido explicativo que el expuesto en el párrafo anterio para la reducción de la velocidad durante la acción. Así el proceso de inhibición de respuestas

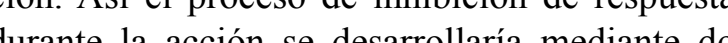
iante do pelacionados [9]. E prinnero inibifa la activación de las posibles respuestas seleccionadas a nivel espinal (control de mpulsos) para evitar salidas prematuras y el segundo tendría que tomar la decisión de continuar con el ataque directo o inhibir éste para cambiar a otra acción asociada (resolución de conflictos). De este modo, antes de iniciar el movimiento, se activarían las dos respuestas posibles, requiriéndose señales inhibitorias a nivel espinal, a la espera de informion exterior que haga prevalecer una de segundo mecanismo inhibitorio ocutiria a niveles corticales superiores, produciend una cierta demora en la respuesta $[10,11]$, lo que nos permite pensar que la incertidumbre debida a posibilidad de error durante la acción de ataque, ncrementaría el tiempo de reacción de los esgrimistas.

A pesar de la cautela que debemos tener al comparar el tiempo de reacción de inicio de movimiento $\left(\mathrm{TR}_{1}\right)$ y el que se produce durante la acción $\left(\mathrm{TR}_{2}\right)$, debida a la diferente sensibilidad de los sistemas de registro utilizados, el hecho de no haber existido diferencias estadísticamente significativas entre el $\mathrm{TR}_{1}$, registrado en la condición CIA y $\mathrm{TR}_{2}$, pone de manifiesto que el movimiento no afectaría al TR y apoyaría las teoría expuesta sobre el efecto de las señales inhibitorias sobre el tiempo de reacción

Las teorías basadas en el modelo teórico sobre as dos corrientes de la percepción visual, [12,13] también confirmarían los resultados expuestos en esta investigación. Así, cuando se realiza una acción sin que se vea afectad fensivas no previtas del oponente, la coriente derive

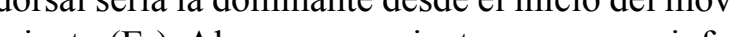
miento $\left(E_{1}\right)$. Al ser una corrente que recoge información visual implicita en el movimiento, permite que el movimiento se realice de forma rápida, automatizada y manteniendo una buena precisión de movimiento hasta alcanzar el objetivo [15]. Por e contrario, cuando existe la posibilidad de erro durante la acción el ataque, la corriente ventral, asociada a la consciencia explícita domina sobre la dorsal La consecuencia es que et movinien se realizaría más lentamente, aunque exista una acción asociada y alternativa al posible error. Investigaciones precedentes indican que cuando accion alternativa al error es imprevista o no esta asociada a la primera acción de ataque, el TR se incrementa y el movimiento se hace aún más lento [6].

\section{Conclusiones}

Cuando existe la posibilidad de error durante 1 realización del ataque en esgrima, se incrementan os parámetros temporales de la respuesta de reacción (TR y TM), la velocidad de ejecución disminuye $y$, en consecuencia, el movimiento se hace más lento. Unos resultados que ponen de manifiesto la importancia que tiene en esgrima la intención táctica para predecir con éxito los movimientos defensivos que realizará el oponente durante acción la accion de atzue. La predicción que se accion la accion de atraue. La prediccion que se cuentemente, la velocidad de ejecución del ataque.

\section{Bibliografía}

1. Gutiérrez-Dávila, M., Rojas, F.J., Antonio, R. y Navarro, E. (2011). Response timing in the lunge and target change in elite versus médiumlevel fencers. European Journal of Sports Sciences, i-Firts, 1-22

2. Schmid, R.A., y Lee, T.D. (2011). Motor contro and learning. (5TH 5th ed.). Champaign, IL: Human Kinetics.

. Stein, J.F. (2008). Factors influencing the initiation, performance and precision of the hit in fencing. In X. Iglesias (Ed.): Proceedings of the 1st International Congress on Science and Techno gy in Fencing. (pp. 7-13), Barcelona, Spain.

4. Borysiuk, Z., \& Waskiewicz, Z. (2008). Information processes, stimulation and perceptual training in fencing. Journal of Human Kinetics, 19 63-82.

. Czajkowski, Z. ( 2009). Tactics in fencing. Preparatory actions. Studies in Physical Culture and Tourism, 16 (4), 371-378

6. Gutiérrez-Dávila, M., Rojas, F.J., Antonio, R. y Navarro, E. (2013). Effect of Uncertainty on the Reaction Response in Fencing. Resea Quarterly for Exercise and Sport, 84, 1, 16-23.
Desmurget, M. y Sirigu, A. (2009). A parietalpremotor network for movement intention an 419

Gao, J., Wong-Lin, K., Holmes, P., Simen, P. y Cohen, J.D. (2009). Sequential effects in twochoice reaction time tasks: Decomposition an synthesis of mechanisms. Neural Comput. 21 (9) 2407-2436.

Duque, J., Lew, D., Mazzocchio, R., Oliver, E. y Ivry, R.B. (2010). Evidence for two concurrent inhibitory mechanisms during response prepara-
tion. J. Neurosci, $30(10): 3796-3802$ tion. J. Neurosci., 30 (10): 3796-3802.

Schluter, N.D., Rushworth, M.F., Passingham R.E. y Mills, K.R. (1988). Temporary interference in human lateral premotor cortex suggests dominance for the selection of movements. A study 121: 785-79.

11. IVant, J. Braning, P. y Mats, R. (2009). Mapping the pathways of information processing from sensation to action in four distinct sensor(12): 4167

12. Goodale M. A. y Westwood, D. (2004). A. An evolving view of duplex vision. separate but intion. Curr Opin Neurobiol, 14: 203-211.

brain in action Oxford: Oxford University Press Glover, JJ (2004). Separate visual rity Presenttions in the planning and control of action. Behavioral and Brain Sciences, 27, 3-78.

15. Van der Kamp, J., Rivas, F., Van Doorn, H. y Savelsbergh, G. (2008).Ventral and dorsal sysem contributions to visual anticipation in fast ball sports. Int. J. Sport Psychol., 39: 100-130.

16. Zatsiorsky, V.M. y Seluyanov, NV (1983) The mass and inertial characteristics of the main segments of the human body. In: Biomechanics VIII-B. Matsui, H. and K. Kobayashi (Eds) Champaign, I.L: Human Kinetics, 1152-1159.

17. Leva, De, P. (1996). Adjustments to ZatsiorskySeluyanovs segment inertia parameters. Journal of Biomechanics. 29(9): 1223-1230.

18. Winter, D. (1990) Biomechanics and Motor Control of Human Movement, Second Edition. Published by John Wiley \& Sons, New York.

19. Sanderson, F.H. (1983). The effect of directional uncertainty on reaction time and movement time in a fencing task. Journal of Sports Sciences, 1 